\title{
riccafd
}

Revista Iberoamericana de Ciencias de la Actividad Física y el Deporte

\section{LA CULTURA DEPORTIVA EN CHILE, META-ANÁLISIS SOBRE HÁBITOS DE ACTIVIDAD FÍSICA \\ Y DEPORTE 2006-2018}

\section{SPORTS CULTURE IN CHILE, META-ANALYSIS ON PHYSICAL ACTIVITY AND SPORTS HABITS 2006-2018}

Bossay Salinas, CA $^{1}$.

${ }^{1}$ Académico adjunto, Escuela de Ciencias del Deporte y Actividad Física, Facultad de Salud, Universidad Santo Tomas, Santiago de Chile. Chile.

claudiobossay@santotomas.cl

\section{Responsabilidades}

${ }^{A}$ Diseño de la investigación

${ }^{B}$ Recolector de datos

${ }^{\mathrm{C}}$ Redactor del trabajo

DTratamiento estadístico

EApoyo económico

FIdea original y coordinador de toda la investigación

Recibido el 28 de junio de 2019

Aceptado el 20 de febrero de 2020

Correspondencia: Claudio Bossay claudiobossay@santotomas.cl

DOI: http://dx.doi.org/10.24310/riccafd.2020.v9i1.8302

\section{RESUMEN}

Este articulo pretende dar algunas nociones de lo que se debería tomar en cuenta al momento de hablar de "cultura deportiva", junto con entregar las principales características de la cultura deportiva chilena. En una primera parte revisaremos los diversos elementos que deben ser considerados a la hora de comprender la expresión "cultura deportiva", para luego exponer antecedentes estadísticos sobre los hábitos y costumbres de los chilenos y chilenas en temáticas de prácticas físicas deportivas de los últimos años, para finalizar con una discusión a modo de conclusiones sobre la cultura deportiva chilena.

Palabras clave: cultura, deporte, prácticas físicas deportivas, hábitos, encuestas. 


\section{ABSTRACT}

This article aims to give some notions of what should be taken into account when talking about "sports culture", along with delivering the main characteristics of the Chilean sports culture. In a first part we will review the various elements that should be considered when understanding the expression "sports culture", to then present statistical data on the habits and customs of Chileans in sports physical practice topics of recent years, to end with a discussion as conclusions about the Chilean sports culture.

Key words: culture, sport, sports physical practices, habits, surveys.

\section{INTRODUCCIÓN Y MARCO CONCEPTUAL}

Las visiones más tradicionales sobre lo que se puede comprender sobre "cultura" nos entregan una mirada amplia sobre el concepto, una visión de mundo que incluye la vida material, intelectual y espiritual, hasta un significado genérico, donde es todo lo creado por los seres humanos, "es ese todo complejo que comprende conocimientos, creencias, arte, moral, derecho, costumbres y cualesquiera otras capacidades y hábitos adquiridos por el hombre en tanto que es miembro de la sociedad"(1).

A estos antecedentes hay que adicionar, que la cultura es algo, que se construye a partir de las mismas personas que son parte de esta misma (cultura) y que cambia con el tiempo y el espacio (lugar), especialmente debido a que esta comprende "conocimientos" y siempre estamos buscando nuevos saberes (2).

Por ende, la cultura es como una pauta que regula el quehacer de los seres humanos que viven en sociedad, pone los límites (valores, costumbres, etc.) y entrega los parámetros de comportamiento. Estas pautas cambian, son moldeables, pero mantiene sus grandes lineamientos que la hacen reconocible y distinguible entre otras culturas.

En esta línea Norbert Elías (3), a fines del siglo pasado, en su intento de comprender a las sociedades cortesanas, nos invita a reflexionar sobre los fenómenos sociales o culturales en el largo plazo, entendiendo su evolución, cómo fueros sus procesos, y qué se esperaba de estos. Llevándonos más a una reflexión sobre los "procesos civilizatorios" extendidos en el tiempo, para una mejor comprensión del polisémico concepto de "cultura".

Por otro lado, cuando hablamos de "deporte", al igual que de "cultura", no se puede hablar de una única concepción, y no podemos ceñirnos estrictamente a lo que podríamos comprender, como producto de una disciplina deportiva que posee, tanto normas (pre-establecidas), con algún tipo de agonismo o luchas, así como el atletismo o el fútbol. 
El fenómeno del "deporte" al igual que "cultura", tenemos que comprenderlo como una construcción humana y en constante mutación. Muchos aun no consideran deportes "el skate" o "el parkour", pero ya el primero de estos va a estar en los próximos Juegos Olímpicos Tokio 2020. Dónde dejaríamos fenómenos como la "zumba" u otras manifestaciones que en el consiente e inconsciente de las personas rutinarias que componen la sociedad son deporte o manifestaciones de este. Por ejemplo, en Chile, existen una serie de federaciones deportivas, que muy difícilmente podrán participar en un Juego Olímpico, máxima expresión del deporte moderno, pero que se encuentran adheridas a su filial nacional.

Pierre Bourdieu y sus seguidores (4) comprenden el fenómeno "deporte" como un espacio social cuya articulación funda normas y da formas de "juegos" o "pasa tiempos", intereses y capitales específicos, así como de estructuras, sistemas de disposiciones particulares, que se encuentran relacionados con distintos campos sociales.

Desde esta mirada y acercándonos a la concepción de cultura deportiva, Sandoval y García (5), la detallan como "conjunto de representaciones y significaciones sociales en torno a la actividad física y el deporte, vale decir, la forma en que se conciben las ideas con que se asocian y la valoración que se otorga".

En esta línea los académicos de las Escuelas de Educación Física de Chile, opinan que cultura deportiva debería comprenderse como un "conjunto de ideas, costumbres, emociones y creencias que refieren e interpretan la forma en que las personas y comunidades desarrollan o practican actividades físicas" (6).

\section{MATERIALES, MÉTODO E INSTRUMENTOS}

Se utilizó un método de carácter descriptivo desde la investigación empírica (7) y con una estrategia de meta-análisis (8), de información disponible en diversas páginas web internacionales, e institucionales de servicios públicos del Estado de Chile:

- Informes de series y bases de datos de Encuestas Nacionales de Hábitos deportivos en la población mayor o igual de 18 años 2006-2018 (9).

- Balances de Gestión Integral (BGI), del Instituto Nacional del Deporte (IND) y del Ministerio del Deporte (MINDEP) 2006-2018 (10).

- Evaluaciones de programas gubernamentales en el área de la actividad física y el deporte de la Dirección de Presupuestos (DIPRES), del Ministerio de Hacienda (11).

- Además de otras encuestas de salud, cultura, y estudios particulares de libre acceso o solicitados vías transparencia.

Solo para fines operacionales, para este articulo y basados en los elementos conceptuales antes descritos, enmarcaremos la "cultura deportiva chilena" en 
base a indicadores de hábitos y valoraciones de las encuestas nacionales en este ámbito desde 2006 a la fecha, en base a las siguientes dos grandes dimensiones:

- Indicadores sobre el interés de las practicas física deportiva de la población chilena en general, la valoración que dan a esta en su tiempo libre y otras percepciones o creencias sobre las actividad física-deportivas y sus distintos actores.

- Indicadores vinculados a que práctica como "deporte o actividad física" de forma regular el o la chileno/a.

\section{RESULTADOS}

En la actualidad 3 de cada 10 chilenos/as realiza alguna práctica física deportiva de forma regular, situación que solo ha tenido modificaciones marginales en la última década, menos de un punto porcentual por año aproximadamente (73.6\%: 2006, 66,2\%: 2018).

Para conocer las características de la cultura deportiva chilena, comenzaremos por identificar la valoración o el interés que declaran los chilenos y chilenas por las prácticas físicas deportivas en general. Donde si bien se logra visualizar un alto interés por la actividad física o el deporte, casi del 80\%, llama la atención, que del interés a la práctica física deportiva efectiva, hay más que un paso, menos del $50 \%$ de los que muestra interés por la actividad física o deportiva, concreta su práctica, lo que se ha ido manteniendo con el tiempo, como se logra visualizar en la Gráfica 1.

\section{Gráfica: 1}

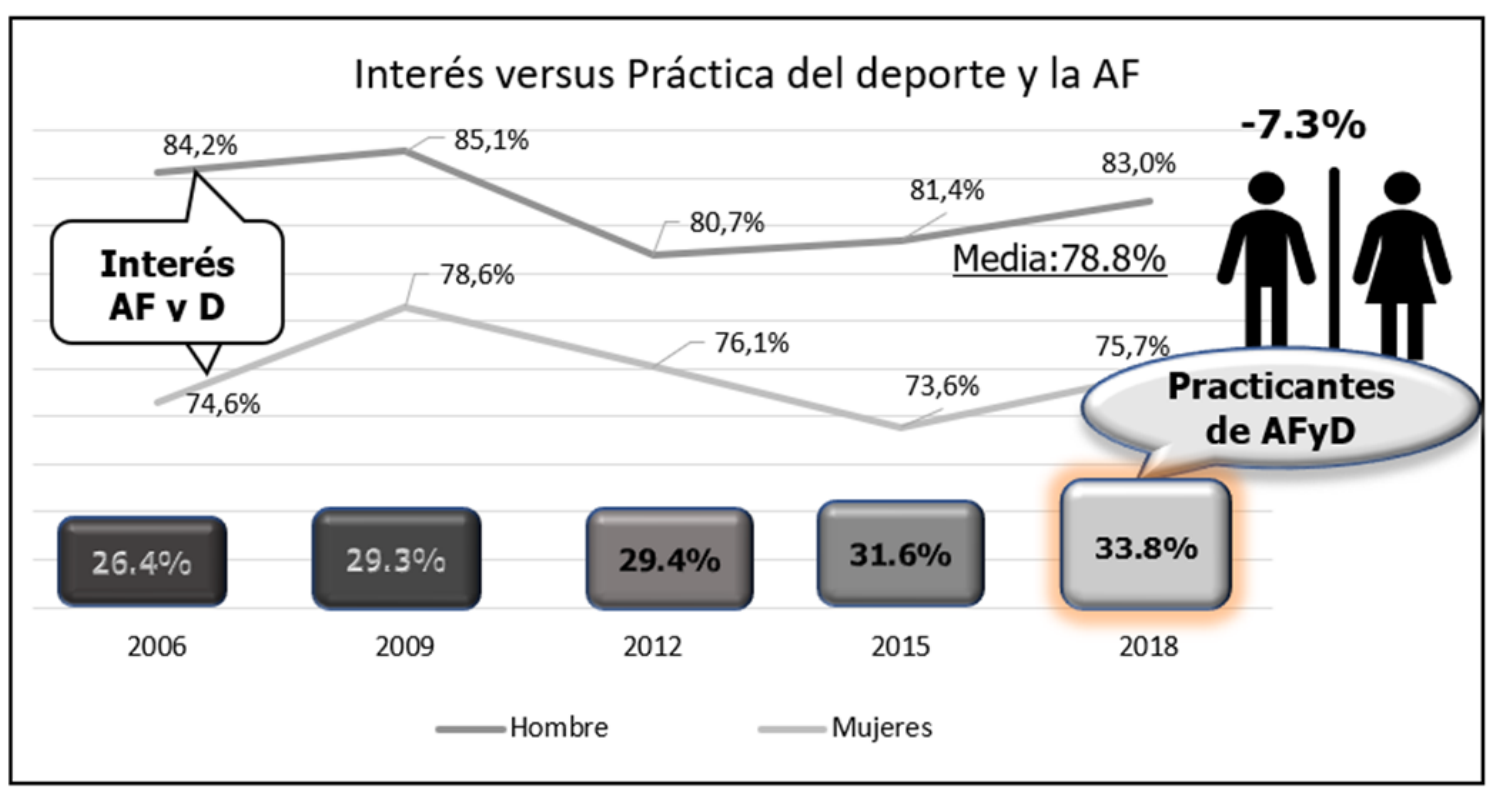

Elaboración propia. 
El interés o no por la práctica física deportiva, posee distinciones por genero al igual que la concreción de este, donde los hombres poseen mayor valoración y práctica que las mujeres.

Desde la utilización del tiempo libre o espacios de ocio de las personas, podemos decir, sobre la base de las mismas encuestas antes citadas, que el practicar deporte o actividad física, si bien ha tenido un aumento en los últimos años, aún no está dentro de las prioridades de las personas (Gráfica 2), prefiriendo más actividades de carácter sedentario, situación que se ve reforzada por la "Encuesta Nacional de Utilización del Tiempo Libre 2015" (EUTL), donde el 90,2\% de las personas prefiere ver televisión, con un promedio de más de dos horas diarias. (2.16 en la semana y 2.51 el fin de semana), y con un aumento en relación con los añ s (12).

\section{Gráfica: 2}

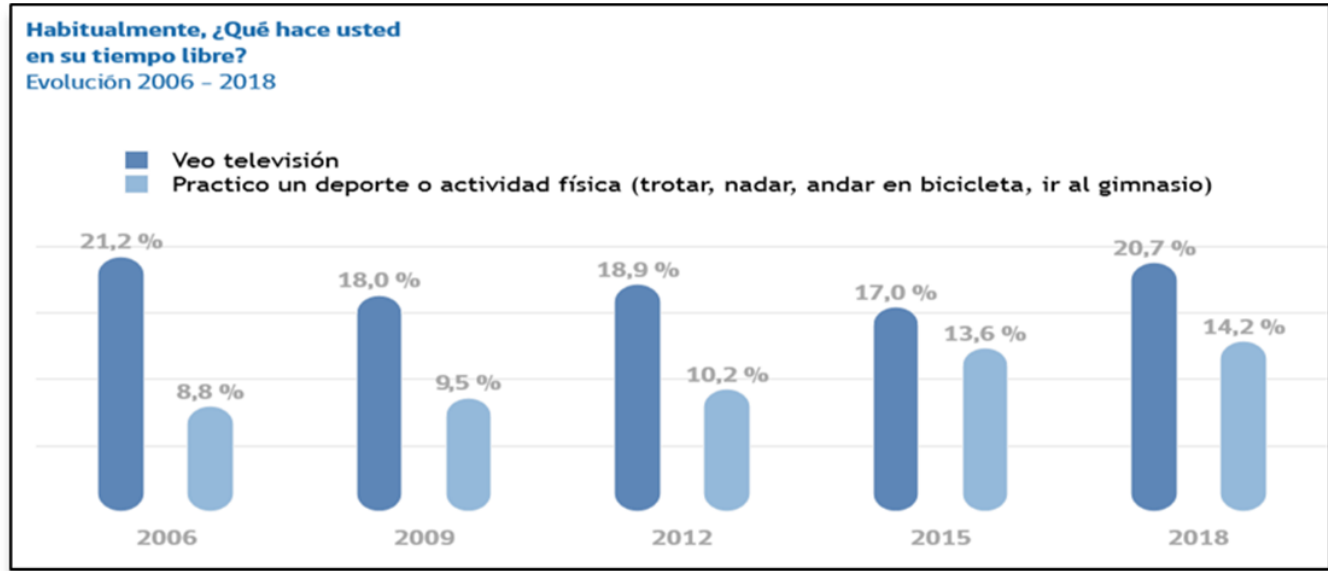

Fuente: http://www.mindep.cl/encuesta-actividad-fisica-y-deporte-2018

En esta misma línea, la base de datos Encuesta Nacional de Televisión 2014 (13), nos revela que, de las personas que "ven televisión" el 75,3 \% (para su primera mención), declara ver programas de tipo "eventos deportivos", siendo los hombres, adultos y adultos mayores de nivel socioeconómico (NSE) medio, y alto los que lideran este indicador como podemos observarlos en el siguiente gráfico

\section{Gráfica: 3}

\section{Ven Evento Deportivo \%.}

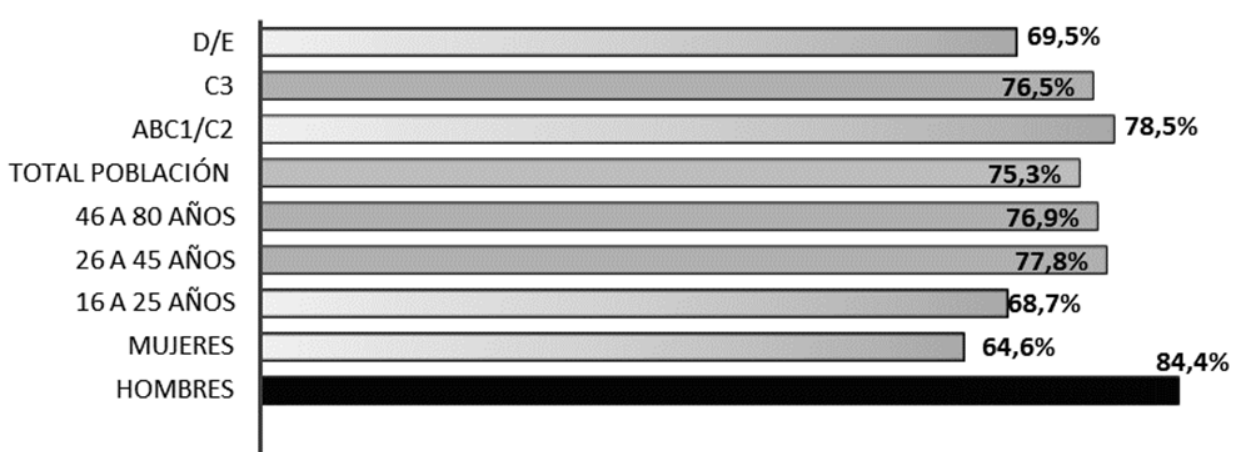

Elaboración propia, sobre la base de datos no ponderados de pregunta. 
Los datos antes expuestos, muestran una alta valoración de las personas por los espectáculos deportivos en la televisión, siendo las mujeres, los jóvenes y los de menos recursos económicos, los que menos ven eventos deportivos, pero siendo siempre este tipo de programa el más mencionado, por muy sobre otros tales como películas, teleseries o noticieros.

Desde las percepciones de los chilenos y chilenas, y en especial sobre su condición física (Tabla 1), esta no ha variado mucho en la última década y en general es buena, solo un $20 \%$ considera que poseen una condición física deficiente o muy deficiente.

Esta percepción contrasta con los indicadores internacionales de la OECD, donde después de Estados Unidos de Norteamérica, Chile es uno de los países con mayor tasa de población obesa con un $34 \%$ en esta situación (14).

Tabla 1

\begin{tabular}{|c|l|c|c|c|c|r|}
\hline $\begin{array}{c}\text { Percepción CONDICIÓN } \\
\text { FISICA }\end{array}$ & $\mathbf{2 0 0 6}$ & $\mathbf{2 0 0 9}$ & $\mathbf{2 0 1 2}$ & $\mathbf{2 0 1 5}$ & $\mathbf{2 0 1 8}$ \\
\hline \multirow{4}{*}{ Muy Buena/ } & Hombres & $42,70 \%$ & $47 \%$ & $\mathbf{4 7 , 8 0 \%}$ & $47,10 \%$ & $48,6 \%$ \\
\cline { 2 - 7 } & Mujeres & $29,90 \%$ & $33,80 \%$ & $34,10 \%$ & $35,60 \%$ & $36,2 \%$ \\
\cline { 2 - 7 } & abc1 & $42,70 \%$ & $\mathbf{4 9 , 8 0 \%}$ & $46,10 \%$ & $\mathbf{4 9 , 8 0 \%}$ & $\mathbf{5 3 , 2} \%$ \\
\cline { 2 - 7 } & $\mathrm{c} 2$ & $37,90 \%$ & $41,00 \%$ & $41,80 \%$ & $43,10 \%$ & $44,5 \%$ \\
\cline { 2 - 7 } & $\mathrm{c} 3$ & $37,80 \%$ & $35,00 \%$ & $36,20 \%$ & $42,10 \%$ & $40,9 \%$ \\
\cline { 2 - 7 } & $\mathrm{d}$ & $34,80 \%$ & $31,00 \%$ & $32,60 \%$ & $36,90 \%$ & $41,5 \%$ \\
\cline { 2 - 7 } & $\mathrm{e}$ & $28,30 \%$ & $29,00 \%$ & $34,20 \%$ & $34,20 \%$ & $36,1 \%$ \\
\hline
\end{tabular}

Elaboración propia. sobre la base de datos no ponderados.

En los últimos 10 años, casi el cien por ciento de los chilenos está de acuerdo o muy de acuerdo con afirmaciones vinculadas más a necesidades sanitarias de la práctica deportiva, tanto para la salud "física" como "mental".

Más diferencias de opiniones existen en otras líneas, como por ejemplo uno de cada cuatro chilenos considera que "Para practicar actividad física o deporte se necesitan condiciones físicas especiales y sobresalientes..." percepción que se atenúa en los niveles socioeconómicos más bajos y con los años, como se logra ver en Gráficas 4 y 5

En esta misma línea, pero particularmente sobre la creencia que se "requiere un gasto importante de dinero" para la práctica física deportiva, también tenemos porcentajes no menores, dentro del $30 \%$, pero que son muchos más homogéneos entre sexo, tramos etarios, y hasta NSE, donde la diferencia entre los NSE solo llega al $10 \%$. 
Gráfica: 4 y 5

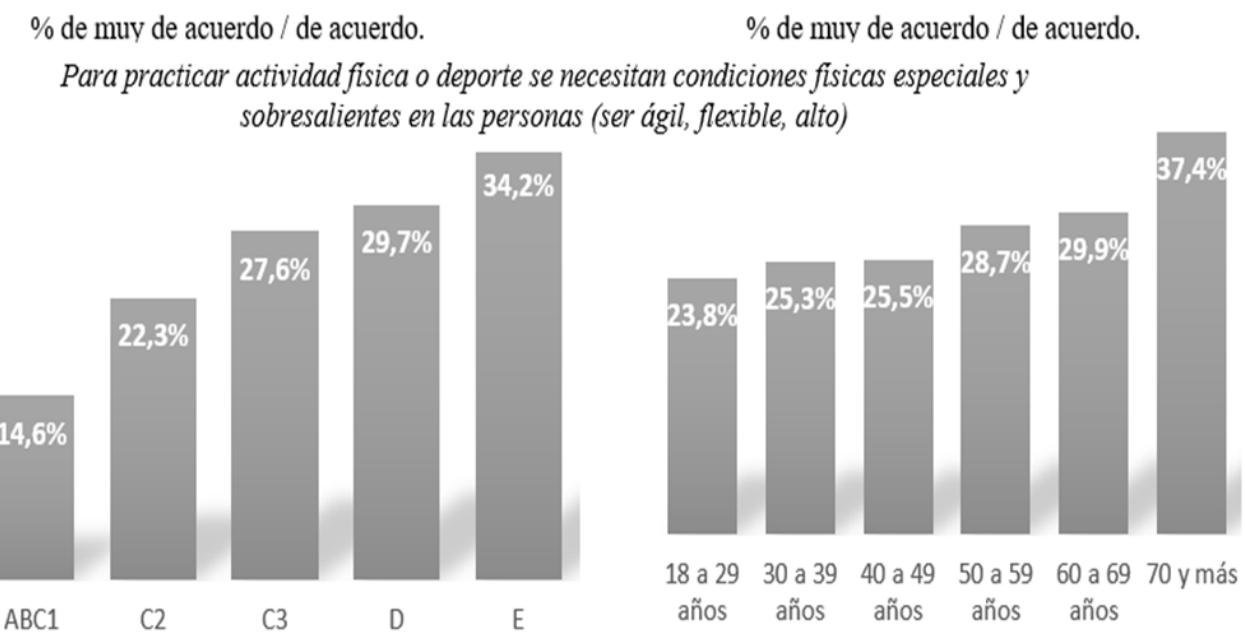

Elaboración propia, datos no ponderados por el total de casos para cada NSE \& tramo etario, y sobre la base de datos del 2015.

Gráfica: 6

$\%$ de muy de acuerdo / de acuerdo.

La práctica de actividad fisica y deportiva requiere un gasto importante de dinero.

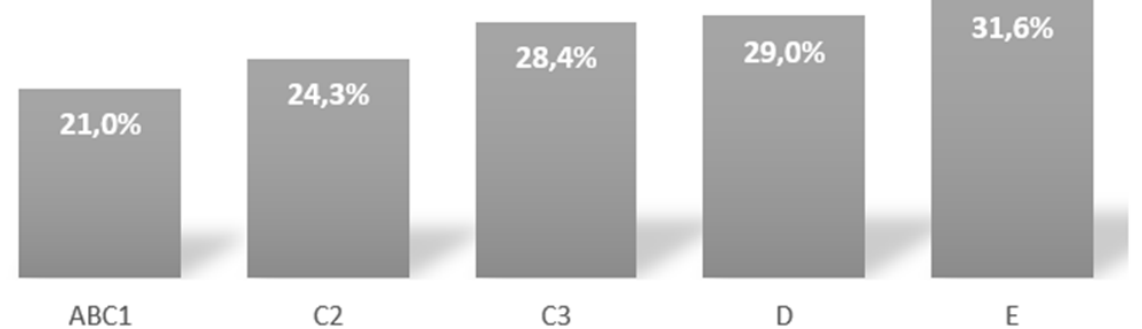

Elaboración propia, datos no ponderados por el total de casos para cada NSE \& tramo etario, y sobre la base de datos del 2015.

Más dispersa es la creencia en otras temáticas, como por ejemplo sobre el rol del Estado o los dirigentes, en el primero de los casos, existe un grupo de la población que considera, que el deporte y la actividad física son tarea y fomento exclusivo del Estado.

Desde la pertenencia a las organizaciones deportivas, en Chile solo el $7 \%$ de la población dice que participa en estas, situación que no ha tenido grandes modificaciones en los últimos años, además los dirigentes de estas organizaciones, han estado mejorando su percepción dentro de la ciudadanía, donde el año 2006, casi un $70 \%$ de la población no tenía una buen imagen de ellos, situación que en la última medición del 2018, esta cifra casi se invierte, con un $60 \%$ de la población que posee una buena percepción de los dirigentes nacionales.

El principal argumento que señala la población nacional para no practicar deporte o física es el "no poseer tiempo" sin cambios significativos en los últimos 
años. Solo una llamativa alza se logra denotar especialmente en la última medición particularmente en la causal "por qué no les gustas" de un estable $10 \%$ en las mediciones anteriores a un $16 \%$ el 2018.

Otro dato que se ha mantenido más o menos estable durante la mayoría de las mediciones, y que dice relación con dato anterior, es que a estas mismas personas que señalan que "no poseen tiempo" para la práctica de actividad física o deporte, cuando se les pregunta, ya no por "sus causales", sino que por las de los otros, causales agrupadas como "la falta de interés, formación y hábitos" supera a la causal de la falta tiempo, como se logra visualizar en gráfica 7

\section{Gráfica: 7}

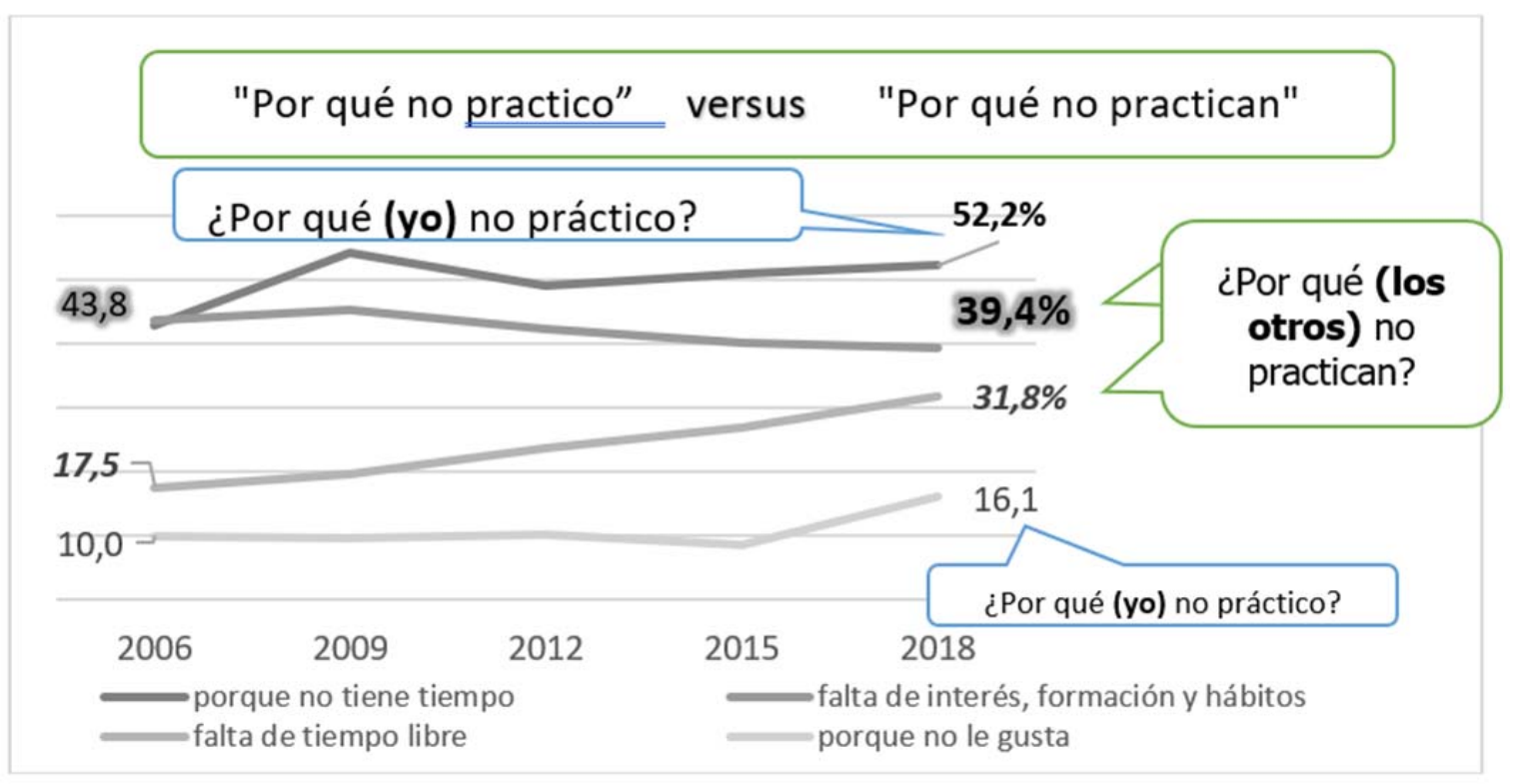

Elaboración propia, datos no ponderados por el total de casos.

Mirado ahora desde lo más formal del concepto deporte, "la norma" y la "competencia", en Chile el futbol y sus derivados tales como baby futbol o futsal han sido por años el deporte más practicado (Tabla 2), especialmente por los hombres, la población más joven y grupos socioeconómicos medios y medios bajos.

Los grupos socioeconómicos con mayor poder adquisitivo, adulto mayor y mujeres poseen otros intereses deportivos, tales como el correr, acondicionamiento físico, baile entretenido o simplemente ir al gimnasio, que en su mayoría son prácticas físicas deportivas con pocas normas y casi nada de competencia o agonismo con otros.

Otras manifestaciones de prácticas físicas deportivas, de carácter colectivo solo marcan datos menores y a la baja con los años, llegando en algunos casos solo a ser datos marginales, nos referimos a disciplinas que son parte de las mallas curriculares de todos los escolares chilenos, tales como el basquetbol o el voleibol, donde la primera de estas en la última medición el 2018 solo marcó un 2,3\%. 
La natación, yoga, aeróbica también bordean el $2 \%$, siendo llamativo el descenso de la aeróbica desde el 2006, cuando ostentaba un $14 \%$ de preferencia de los encuestados.

El correr o "running" parece que ya tuvo su pick el 2015 , con un $17 \%$ de la población que se manifestó por su práctica, manteniéndose estable entorno al $10 \%$ en la actualidad.

El ciclismo, o el andar en bicicleta viene mostrando un acenso sostenido en todas las mediciones, pasando de un $8.3 \%$ el 2006 y llegando a un $14.4 \%$ en la medición del 2018.

Tabla 1

\begin{tabular}{|c|l|c|c|c|c|c|}
\hline $\mathrm{N}^{\circ}$ & $\mathbf{2 0 0 6}$ & $\mathbf{2 0 0 9}$ & $\mathbf{2 0 1 2}$ & $\mathbf{2 0 1 5}$ & $\mathbf{2 0 1 8}$ \\
\hline 1 & Fútbol y similares & $34,3 \%$ & $30,6 \%$ & $\mathbf{2 4 , 3} \%$ & $\mathbf{2 1 , 6 \%}$ & $19,3 \%$ \\
\hline 2 & Corre, running y similares & $11,9 \%{ }^{\star}$ & $9,8 \%$ & $10,3 \%$ & $17,0 \%$ & $9,4 \%$ \\
\hline 3 & Acondicionamiento fisico, gimnasia, y similares & $6,8 \%$ & $16,0 \%$ & $11,2 \%$ & $16,6 \%$ & $13,7 \%$ \\
\hline 4 & Ciclismo, freestyle, y similares & $8,3 \%$ & $8,8 \%$ & $12,1 \%$ & $12,3 \%$ & $14,4 \%$ \\
\hline 5 & Baile,baile entretenido, y similares & $1,4 \%$ & $2,7 \%$ & $5,6 \%$ & $6,6 \%$ & $10,3 \%$ \\
\hline 6 & Caminar, trekking y similares & $\mathrm{S} / \mathrm{M}$ & $4,7 \%$ & $5,0 \%$ & $3,5 \%$ & $7,3 \%$ \\
\hline 7 & Natación y similares & $2,6 \%$ & $2,2 \%$ & $2,1 \%$ & $2,8 \%$ & $2,4 \%$ \\
\hline 8 & Yoga,Chi kung, y similares & $1,6 \%$ & $2,6 \%$ & $2,9 \%$ & $2,5 \%$ & $2,9 \%$ \\
\hline 9 & Tenis & $3,4 \%$ & $2,3 \%$ & $1,5 \%$ & $2,4 \%$ & $1,2 \%$ \\
\hline 10 & Aeróbica, aerobox y similares & $14,0 \%$ & $6,2 \%$ & $7,0 \%$ & $2,1 \%$ & $2,5 \%$ \\
\hline 11 & Basquetbol & $3,7 \%$ & $3,6 \%$ & $2,1 \%$ & $1,5 \%$ & $2,3 \%$ \\
\hline 12 & Otras prácticas & $12,0 \%$ & $10,4 \%$ & $16,0 \%$ & $11,1 \%$ & $14,4 \%$ \\
\hline
\end{tabular}

*Caminar" precodificado en "correr" solo año 2006

Elaboración propia, de respuesta múltiple y no ponderados.

También se puede observar que el porcentaje de otras prácticas física deportivas, ha tenido un aumento con los años, donde se logra pesquisar prácticas físicas deportivas con baja mención, pero agrupables, tales como: las vinculadas a deportes acuáticos como buceo, surf, kayak o remo; u otros vinculados a las ruedas, como el patinaje o skateboad, sumado a algunas actividades físicas de pueblos originarios.

\section{DISCUSIÓN A MODO DE CONCLUSIONES}

Luego de los antecedentes expuestos, podríamos decir que la cultura deportiva chilena, está llena de ambigüedades, por ejemplo: la gran cantidad de los chilenos y chilenas les interesa la practica física deportiva, pero solo la mitad de éstos los practica efectivamente de forma regular.

Poseemos una buena o muy buena percepción de nuestra condición física, también consideramos que realizar deporte o actividad física, es bueno para la salud, la mayoría considera que no es necesario tener condiciones especiales 
o dinero para practicar deporte o actividad física, pero somos líderes en sobre peso y obesidad en América Latina, y solo un 33\% de la población chilena practica deporte o actividad física de forma regular.

El interés por el deporte parece tener una versión más pasiva en chilenos y chilenas, preferimos ver televisión que hacer deporte o actividad física en nuestro tiempo libre, situación que nos ha acompañado por años, pero cuando detallamos que vemos en la Televisión, aparece una nueva faceta de nuestra cultura deportiva. Somos consumidores de espectáculos deportivos, vemos deporte por la TV u otros medios digitales, siendo los hombres de nivel socioeconómico más altos, los que más consumen deporte por la televisión, situación que va de la mano con sus niveles de interés y práctica efectiva de deporte.

Menos ambiguo, parece ser el tipo de deporte o práctica física deportiva que realizamos los chilenos y chilenas. Donde si bien la disciplina fútbol y similares ha sido por años el deporte más practicado en Chile, en las últimas mediciones, se ha notado una tendencia a la baja de éste, llegando al $19 \%$ en la última medición del 2018. Además, se logra visualizar el avance de otras prácticas físicas deportivas, con menos normas, de carácter más libre y menos disciplinar o vinculado a un deporte formal propiamente tal, tales como "correr", "running", "andar en bicicleta" o "caminar" entre otras.

Otro elemento relevante, es la poca formalidad o asociatividad vinculada con el deporte, sumado a una irregular percepción de sus dirigentes, y con más de un tercio de los chilenos y chilenas, que espera que el Estado se haga cargo de sus necesidades. Importante aquí señalar que el ente rector del deporte en Chile, el Ministerio del Deporte en conjunto con el IND, no logra abarcar con sus programas ni un 10\% de la población total nacional (BGI 2018).

Como conclusión final, podríamos decir que la cultura deportiva chilena, no ha tenido grandes cambios en los últimos años, donde somos un país de nos interesa y sabemos que el deporte y la actividad física regular nos hace bien, pero preferimos que otros los hagan, o verlo por la televisión o alguno otro medio digital.

Los que practican, en su mayoría realizan fútbol o algún derivado, pero cada vez son menos estos. Y cada vez más, estamos realizando ejercicios físicos poco regulados y de carácter individual, con una baja o casi nula participación en organizaciones deportivas, esperando que otros solucionen nuestras necesidades deportivas.

Por último, es importante considerar, que hay grupos dentro de la sociedad chilena que poseen diversas percepciones, prácticas y creencias sobre la actividad física y el deporte. Por ejemplo, las mujeres poseen un menor interés por las prácticas físicas deportivas en general, pero una mayor dispersión de estas, no existiendo una práctica hegemónica. Por su lado, los hombres muestran una mayor disposición hacia la práctica, pero menor dispersión en el tipo de prácticas físicas deportivas, practicando especialmente fútbol o algunos de sus derivados. 
Estas distinciones, también pueden expresarse por nivel socioeconómico, y otras características de la población nacional, las cuales sería prudente poder detallar en otras investigaciones.

\section{REFERENCIAS BIBLIOGRÁFICAS}

1. Harris, M. (2011) "Antropología cultural”, Madrid, Alianza Editorial.

2. Barrera, R. (2013). "El concepto de la Cultura: definic ones, debates y usos sociales". Revista de Claseshistoria Artículo Nº 343, 15 de febrero de 2013.

3. Toledo, F (2015) "La teoría de las configuraciones sociales de Norbert Elias y su aplicación a la sociología del deporte recreativo en las nuevas élites de prestigio" Volumen 12, número 28, mayo-agosto, 2015, pp. 215-239 doi: 10.29092/uacm.v12i28.40 .

4. Sánchez, R. (2015) “La contribución de Pierre Bourdieu al estudio social del deporte" EMPIRIA. Revista de Metodología de Ciencias Sociales. N. ${ }^{\circ} 30$, enero-abril, 2015, pp. 161-180. ISSN: 1139-5737. DOI/empiria.30.2015.13889.

5. Sandoval, Pablo y Iñigo García (2014). "Cultura deportiva en Chile: desarrollo histórico, institucional actual e implicancias para la política pública” polis 13(39):441-62 doi: 10.4067/s0718-65682014120000020.

6. Ministerio del Deporte, Gobierno de Chile, (2016). "Política Nacional de Actividad Físicas y Deporte" 2016-2025" (1ª , ed), Santiago de Chile. En: http://www.mindep.cl/ quienes-somos/politica-nacional(2018, 05 diciembre).

7. Heinemann, K. (2003).Introducción a la metodología de la investigaciones empírica, en las ciencias del deporte, edit . Paido tribo.

8. Tomas, J. y Nelson, J. (2007) Métodos de investigación en actividad física" Edit, Paido Tribos Barcelona.

9. Dirección de presupuestos, Ministerio de Hacienda, Gobierno de Chile. Balances de Gestiones Integrales 2018 (BGls) del Ministerio del Deporte y del IND. En www. dipres.cl, (2019, 20 enero.

10. IND-Alcalá Consultores (2015) Informe final. "Encuesta Nacional de Hábitos de Actividad Física y Deportes 2015 en la Población de 18 años y más".

11. Ministerio del Deporte, Gobierno de Chile, (2016). "Encuesta Nacional de Hábitos de Actividad Física y Deporte 2018 en Población de 18 años y más" Resumen ejecutivo en: http://www.mindep.cl/encuesta-actividad-fisica-y-deporte-2018/, (2018, 05 diciembre).

12. Instituto Nacional de Estadísticas (2016) Informe Encuesta Nacional de Utilización del Tiempo Libre 2015, en: https://www.ine.cl/estadisticas/menu-sociales/enut, (2018, 20 diciembre).

13. Consejo Nacional de la Televisión de Chile, (2014). $8^{\circ}$ Encuesta Nacional de Televisión, en: www.cntv.cl/viii-encuesta-nacional-de-television-2014/cntv/2015-1209/124713.html (2019, 01 Abril).

14. Organización para la Cooperación y el Desarrollo Económicos (OECD) (2017), Informe de actualización de la Obesidad de la en: http://www.oecd.org/health/healthsystems/Obesity-Update-2017.pdf (2019, 10 enero). 